\title{
Mapping of Quantitative Trait Loci for Fusarium Head Blight Resistance in Barley
}

\author{
Zhengqiang Ma, Brian J. Steffenson, Louis K. Prom, Nora L. V. Lapitan
}

\begin{abstract}
First and fourth authors: Department of Soil and Crop Sciences, Colorado State University, Ft. Collins 80523; second author: Department of Plant Pathology, North Dakota State University, Fargo 58105; and third author: Department of Plant Pathology, University of Arkansas, Fayetteville 72701.
\end{abstract}

Current address of Z. Ma: Agronomy Department, Nanjing Agricultural University, Nanjing, Jiangsu 210095. P.R. China. Accepted for publication 12 June 2000.

\section{ABSTRACT}

Ma, Z., Steffenson, B. J., Prom, L. K., and Lapitan, N. L. V. 2000. Mapping of quantitative trait loci for Fusarium head blight resistance in barley. Phytopathology 90:1079-1088.

Fusarium head blight (FHB) is a devastating disease that causes significant reductions in yield and quality in wheat and barley. Barley grains infected with deoxynivalenol (DON), a vomitoxin produced by Fusarium graminearum, are rejected for malting and brewing. Among six-rowed barley cultivars tested thus far, only cv. Chevron exhibited resistance. This study was conducted to map genes and to identify DNA markers for marker-assisted breeding for FHB resistance in cv. Chevron with restriction fragment length polymorphism (RFLP) markers. A doubled haploid (DH) population was created from a cross between cv. Chevron and susceptible cv. Stander. Seven field experiments were conducted in four different locations in 2 years. A RFLP map containing 211 loci and covering over 1,000 centimorgans (cM) of

Fusarium head blight (FHB) or scab is a devastating disease of both wheat and barley in many countries with temperate climates $(19,33)$. In the Upper Midwest region of the United States, FHB has emerged as the most important disease of wheat and barley over the past 7 years (1993 to 1999) (17,27). The disease is caused primarily by Fusarium graminearum and can result in yield reductions, decreased market value of the grain, poor seed quality, and lowered seed germination (16). Additionally, the fungus produces deoxynivalenol (DON), a mycotoxin that is toxic to humans and other animals $(6,24)$ and compounds and causes overfoaming or gushing in beer (23). To avoid quality control problems, some malting and brewing companies will not purchase barley with detectable levels of DON (27). The widespread and severe epidemics of FHB over the past 7 years have left much of the Upper Midwest barley crop unsuitable for malting and brewing (27).

$F$. graminearum overwinters on infected residues of host crops such as wheat, barley, corn, and other plant species (19). The recent epidemics of FHB in the Upper Midwest were due to the widespread cultivation of susceptible cultivars, an abundance of pathogen inoculum in the field from the practice of minimum tillage, and unusually high precipitation during the period when the crops were heading $(17,27)$.

The most effective strategy for controlling FHB of barley is through the deployment of resistant cultivars (27). All of the major malting barley cultivars that are currently grown in the Upper

Corresponding author: N. Lapitan; E-mail address: nlapitan@1amar.colostate.edu

Publication no. P-2000-0731-01R

(C) 2000 The American Phytopathological Society the genome was used to map quantitative trait loci (QTL) associated with relatively low FHB severity and DON concentration. Morphological traits differing between the parents were also measured: heading date, plant height, spike angle, number of nodes per $\mathrm{cm}$ of rachis in the spike, and kernel plumpness. Many of the QTL for FHB and DON coincided with QTLs for these morphological traits. The "fix-QTL" algorithm in Mapmaker QTL was used to remove the part of the variance for FHB resistance that may be explained by heading date or plant height. Results from this study suggest that QTLs with major effects for FHB resistance probably do not exist in cv. Chevron. Three QTL intervals, Xcmwg706-Xbcd441 on chromosome 1H, Xbcd307b-Xcdo684b on chromosome $2 \mathrm{H}$, and $X c d o 959 b-X a b g 472$ on chromosome $4 \mathrm{H}$, that are not associated with late heading or height may be useful for markerassisted selection.

Additional keywords: Hordeum vulgare, scab.
Midwest are susceptible. Resistance has been identified in a number of two-rowed barley lines $(22,29,31)$; however, six-rowed cultivars are the preferred type for malting in the region. Unfortunately, very few six-rowed lines have been identified with useful levels of FHB resistance. Cv. Chevron (CI 1111) is the most resistant six-rowed barley identified to date. Its resistance was first discovered in the 1930s by Shands (25) after extensive germ plasm screenings for FHB resistance in Madison, Wisconsin. The FHB resistance of cv. Chevron was confirmed in several recent studies $(2,22)$. Moreover, this cultivar accumulates low levels of DON under epidemic conditions. This latter factor is critical, because the level of DON in barley is now one of the most important quality concerns for the malting and brewing industries.

The evaluation of breeding lines for resistance to FHB and the accumulation of mycotoxins (specifically DON) is a timeconsuming, labor intensive, and expensive process (27). Studies designed to determine the number and chromosomal location of loci contributing to FHB resistance and the accumulation of DON are urgently needed for the resistance breeding effort. This information can be obtained most efficiently by the development of a molecular marker map made from a population segregating for FHB resistance. The construction of such a map could lead to the development of a molecular marker-assisted FHB resistance breeding program. This type of breeding scheme is ideal for diseases like FHB because phenotyping is difficult and expensive.

The objective of this study was to map loci conferring resistance to FHB and the accumulation of DON in the six-rowed cv. Chevron. The chromosomal location of morphological and agronomic traits (e.g., spike angle, heading date, etc.) also was determined to study their possible effect on FHB development. 


\section{MATERIALS AND METHODS}

Plant materials. A single plant selection of the FHB resistant cv. Chevron was used as the female in a cross with cv. Stander (PI 564743), a susceptible malting barley cultivar from the Upper Midwest. One hundred forty-seven doubled haploid (DH) progeny were produced from cvs. Chevron-Stander $F_{1}$ plants using the Hordeum bulbosum technique (1). These DH progeny were provided by P. M. Hayes at Oregon State University, Corvallis.

Disease assessment and mycotoxin assays. Parents and $\mathrm{DH}$ progeny were assessed for FHB severity (in percent) in seven environments at four different locations in 1996 and 1997: Langdon, North Dakota in both 1996 (environments L96a and L96b) and 1997 (L97a and L97b), Fargo, North Dakota in 1997 (F97), St. Paul, Minnesota in 1996 (StP96), and Hangzhou, China in 1997 (H97). DON accumulation (in parts per million) was also measured in all environments, except StP96. A randomized complete block design with two replicates each was used in environments L96a and StP96 with the parents and 143 and 90 DH lines, respectively. Entries in these environments were planted in hill plots (10 to 20 seeds) spaced $0.33 \mathrm{~cm}$ apart in two adjacent rows. The planting for environment L96b, using the same $143 \mathrm{DH}$ lines as in L96a, was made 14 days after the planting for environment L96a and without replication. The same $143 \mathrm{DH}$ lines also were used for field trials in F97 with two replications, and in nonreplicated field trials in L97a and L97b. One hundred and forty-one $\mathrm{DH}$ lines were evaluated in a nonreplicated field trial in H97. In 1997, all entries were planted in 1.0-m rows. The omission of DH lines from some field experiments was due to low seed amounts. Planting, maintenance of plots, and inoculation protocols were followed as described by Prom et al. (22). Four group two isolates of $F$. graminearum (KB-171, KB-172, KB-173, and KB-176) were used as inoculum in the North Dakota nurseries. Local isolates were used in St. Paul and in China. Disease assessments were made when the parents and individual $\mathrm{DH}$ lines were at the mid-dough stage of development (growth stages 84 to 86) (34). The percent severity of FHB was determined by counting the number of infected kernels (those with greater than one-fourth of their surface area showing disease symptoms) and dividing that quantity by the total number of kernels in that spike multiplied by 100 (22). These assessments were made on 10 to 20 randomly selected spikes per plot as described by Prom et al. (22). When the plants were mature, all spikes from each plot were harvested, dried, threshed, and cleaned. DON assays were made according to the method developed by Tacke and Casper (28). For this assay, a random 6-g sample of seed was used from each parent and DH line (21). DON assays were not made from plants in the StP96 environment.

Morphological and agronomic trait assessment. Various morphological (especially spike characters) and agronomic traits may affect the development of FHB on plants in the field (27). To determine the possible contribution of such factors on FHB severity, assessments were made on heading date (environments L96a, L96b, StP96, L97a, L97b, F97, and H97), plant height (L96a, L97a, L97b, F97, and H97), spike angle (F-GH98; GH refers to greenhouse), kernel plumpness (F-GH98), and the number of nodes per $\mathrm{cm}$ of rachis in the spike (kernel density; F-GH98). Heading date was defined as the number of days from planting to when $50 \%$ of the plants in a plot had emerged spikes. Plant height was the number of centimeters from the ground to the tip of the spike, excluding the awns. Spike angle and the number of nodes per centimeter of rachis were determined on the parents and a set of $150 \mathrm{DH}$ lines (143 lines were the same as those used for measuring FHB severity and DON accumulation) grown under the controlled conditions of the greenhouse in 1998. Spike angle was rated at maturity on a scale of 1 to 3 : spikes bending less than $45^{\circ}$ from vertical were scored as 1 ; spikes bending from 45 to $120^{\circ}$ from vertical were scored as 2; and spikes bending greater than $120^{\circ}$ from vertical were scored as 3 . The number of nodes per centimeter of rachis was measured on four randomly selected spikes for each parent and DH line. Kernel plumpness (size) also was assessed in the DH population to determine whether FHB resistance from cv. Chevron, with extremely thin kernels, could be combined with the plump kernel character of cv. Stander. Kernel plumpness is an important quality factor in malting barley and was defined as the percentage (by weight) of a 10-g sample retained on a $2.38 \times 19.05 \mathrm{~mm}$ slotted sieve after shaking for $30 \mathrm{~s}$ on a Niagra sample grader (S. Howes Co. Inc., Silver Creek, NY). This character was assessed on plants grown in the greenhouse (FGH98) to avoid the possible confounding effects of disease on kernel size.

Construction of a framework molecular marker map. DNA extraction and Southern blot analysis were followed as described by $\mathrm{Ma}$ and Sorrells (15). Restriction fragment length polymorphism (RFLP) markers previously mapped in barley and wheat were surveyed for polymorphisms between cvs. Chevron and Stander. These markers included the cMWG and MWG (4), ABC and ABG (8), BCD, CDO and WG (5), BG (26) (N. Lapitan, unpublished data), and KSU (3) sets. Polymorphic markers were mapped using a population of $147 \mathrm{DH}$ lines. Then, a RFLP map was constructed with MAPMAKER 3.0 (Whitehead Institute, Cambridge, MA) program (11). A $\log _{10}$ odds ratio (LOD) score of 3.0 was used to determine the order of the loci. Mapmaker 2.0 for the Macintosh computer was used for drawing the map.

Barley chromosomes were designated as $1 \mathrm{H}, 2 \mathrm{H}, 3 \mathrm{H}, 4 \mathrm{H}, 5 \mathrm{H}$, $6 \mathrm{H}$, and $7 \mathrm{H}$ in the Triticeae system (13). These chromosome numbers correspond to the original barley chromosome designations of $5,2,3,4,7,6$, and 1 , respectively.

Statistical analysis and quantitative trait loci mapping. Statistical analysis was conducted using Data Desk software program (Version 5; Data Description Inc., Ithaca, NY). Data for FHB severity and DON concentration for $65 \mathrm{DH}$ lines in the L97b environment were excluded from the analysis due to lodging. Lodged plants retain moisture longer than unlodged plants and exhibit unusually high levels of FHB and DON (B. Steffenson, unpublished data). Data from different environments were analyzed separately. Heritability was estimated based on entry means using the formula $h^{2}=\sigma_{\mathrm{g}}{ }^{2} /\left(\sigma_{\mathrm{g}}{ }^{2}+\sigma_{\mathrm{e}}{ }^{2} / r\right)$, where $\sigma_{\mathrm{g}}{ }^{2}$ and $\sigma_{\mathrm{e}}{ }^{2}$ are the genotypic and error variance, respectively, and $r$ is the number of replications. Pearson correlation coefficients were calculated to assess phenotypic correlation among traits.

Mapmaker quantitative trait loci (QTL) 1.1 was used for QTL localization $(12,20)$. Although composite interval mapping programs are available and have been reported to detect more QTLs (14), we used Mapmaker/QTL because our goal was to obtain a conservative estimate of the most important QTLs (i.e., large effect QTLs) and their chromosomal positions. Mapmaker/QTL also allows detection of additional QTLs not previously detected using the entire map through the "fix QTL" algorithm (described below). A LOD score of 2.0 was used as the threshold for declaring the presence of a QTL, which is equal to an overall significance level of 0.05 in a "sparse map" case (10). The variance explained by a QTL was calculated by the "MAP" command of Mapmaker/ QTL with the map locations of the QTL peaks as the sequence. Mapmaker/QTL was also used to determine the map positions of confidence intervals surrounding each peak, i.e., the boundaries indicating the width of the peak before the LOD score drops to 1 .

When there was more than one likelihood peak on the same chromosome, the "fix QTL" algorithm of Mapmaker/QTL was used to determine whether the multiple peaks were caused by one or several QTLs (10). This was done by picking the single most likely QTL based on the LOD score, fixing it in place, and rescanning the genome for other QTLs. QTLs identified in the second-pass scanning are shown in the tables and in Figure 1.

When evidence exists that some part of a phenotypic variance can be explained by another phenotype, the effect of the associ- 
ated phenotype can also be fixed in Mapmaker/QTL to identify other QTLs for the trait in question (M. Daly, personal communication). Specifically, this analysis was carried out by setting the sequence as "trait", where trait is heading date or height followed by the "scan" command (11). Since heading date and height could affect the low level of FHB in DH lines, second-pass scanning of the genome for FHB QTLs was performed when a significant correlation existed between FHB severity and heading date or height after fixing heading date or height as a covariable. The QTLs obtained from this analysis are referred to as "secondary" QTLs in this study and are shown with two asterisks in Figure 1. The LOD threshold for this analysis was arbitrarily set at a value 1.0 higher than the log-likelihood achieved by mapping only the covariable like a QTL.

\section{RESULTS}

Assessment of disease, morphological, and agronomic traits. Table 1 shows the means of the parents and the means and ranges of the DH lines for FHB severity, DON concentration, heading date, height, kernel plumpness, spike angle, and number of nodes per centimeter of rachis in the spike across the different environments.

FHB severity. Cv. Chevron (overall mean: $4.0 \%$ ) exhibited a markedly lower level of FHB severity than cv. Stander (overall mean: $31.1 \%$ ) in all environments. The difference in magnitude for FHB severity between the parents ranged from $\approx 4 \times$ in the
L96a environment to almost $70 \times$ in the L96b environment. The DH lines exhibited a range of FHB severity values that were close to those observed for the mean of FHB severity values of the two parents. Several DH lines in environments L96a and H97 exhibited FHB severity levels that were much higher than those observed for cv. Stander. FHB severity showed a near normal distribution in environment L96a (Fig. 2A), whereas in all other environments the distribution skewed toward the resistant parent (data not shown). The continuous distribution of values for FHB severity in the DH population indicates quantitative inheritance for this trait. Heritability for FHB severity was estimated at $31 \%$ based on data from L96a, the only environment that had replicated experiments.

DON levels. Cv. Chevron (overall mean: 4.7 ppm) exhibited markedly lower DON concentrations than cv. Stander (overall mean: $54.2 \mathrm{ppm}$ ) in all environments as was found for FHB severity. The difference in magnitude for DON concentration between the parents ranged from over $8 \times$ in the L97b environment to over $26 \times$ in the F97 environment. In the majority of environments, the DON concentration for several DH lines fell outside the means of the two parents. The frequency distribution of DON concentration in six environments where measurements were taken skewed toward cv. Chevron (Fig. 2B). As with FHB severity, the continuous distribution in values for DON content in the $\mathrm{DH}$ population indicates quantitative inheritance for this trait. The standard deviation for DON concentration was much larger than that
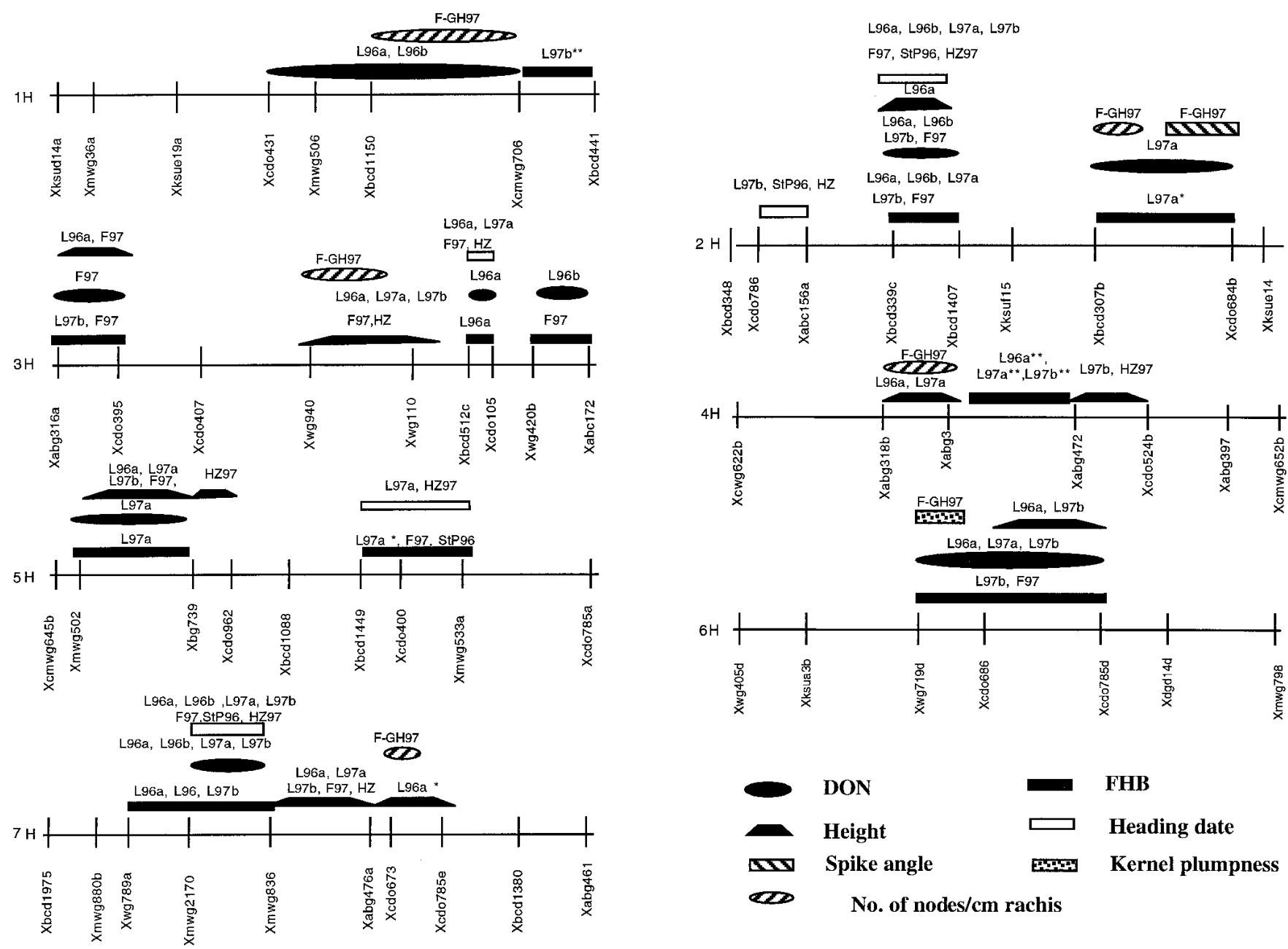

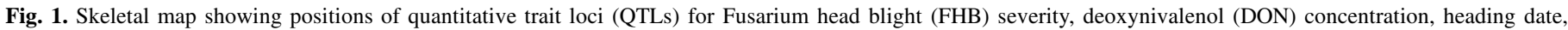

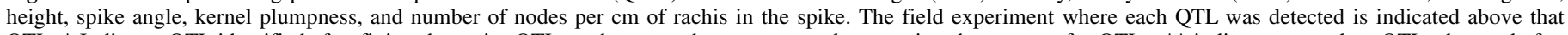

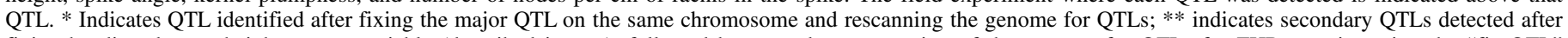

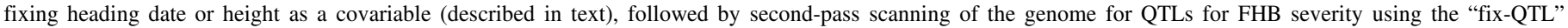
algorithm in Mapmaker/QTL (map is not drawn to scale.). 
for FHB severity in all environments, except L96b and H97 (Table 1). Heritability for DON concentration was estimated at $24.8 \%$ based on data from the replicated experiments in environment L96a.

Heading date and plant height. $\mathrm{Cv}$. Chevron headed later (overall mean difference of $\approx 4$ days) and was markedly taller (mean difference of $\approx 26 \mathrm{~cm}$ ) than $\mathrm{cv}$. Stander (Table 1). Heading date for the DH population displayed a multipeak frequency distribution in all environments (data not shown) suggesting that this trait is controlled by a few major genes. Plant height displayed a nearly normal frequency distribution in the DH population with the mean lying at the parental midpoint value (data not shown). Heritability values for heading date and plant height were 92.3 and $47.3 \%$, respectively, using data from the L96a environment.

Spike angle and number of nodes per centimeter of rachis. $\mathrm{Cv}$. Stander (spike angle score: 1) has an erect to semi-erect spike, whereas cv. Chevron (spike angle score: 3 ) has a nodding spike that frequently bends downward to the peduncle. The mean spike angle of the DH population was almost exactly midway between the values for the two parents (spike angle score: 2.1 ) and the range was from 1 to 3 . The average number of nodes per centimeter of rachis was similar for cvs. Stander (7.49) and Chevron (7.78). A wide range of values (between 3.2 and 9.1) was observed among the DHs, with a mean (7.32) slightly lower than that of the parents.

Kernel plumpness. The agronomically advanced cv. Stander (overall mean: $96.4 \%$ plump) had much plumper kernels than cv. Chevron (21.8\% plump). The mean percentage of plump kernels in the DH population (79.2) was closer to that of cv. Stander than cv. Chevron.

TABLE 1. Means of cvs. Chevron and Stander and the doubled haploid (DH) lines for Fusarium head blight severity (FHB; given as \% of infected kernels per spike), deoxynivalenol (DON) concentration of harvested grain (in parts per million), heading date (HD; days after planting), height (HT; in centimeters), kernel plumpness (KP; in grams), spike angle (SA), and number of nodes per centimeter of rachis $(\mathrm{NN})$

\begin{tabular}{|c|c|c|c|c|c|c|}
\hline \multirow[b]{2}{*}{ Trait } & \multirow[b]{2}{*}{ Test site } & \multirow[b]{2}{*}{ Chevron } & \multirow[b]{2}{*}{ Stander } & \multicolumn{3}{|c|}{ DH } \\
\hline & & & & Means ${ }^{2}$ & Range & Standard error \\
\hline \multirow[t]{7}{*}{ FHB } & L96a & 12.0 & 49.0 & 35.9 & $11.3-60.8$ & 0.71 \\
\hline & L96b & 0.3 & 20.9 & $4.4^{*}$ & $0.2-17$ & 0.28 \\
\hline & St.P96 & 1.6 & 14.1 & $3.7 *$ & $0.8-13.0$ & 0.22 \\
\hline & L97a & 7.3 & 54.0 & $20.2 *$ & $7.6-53.2$ & 0.92 \\
\hline & L97b & 1.6 & 25.6 & $7.9^{*}$ & $0.8-33.3$ & 0.43 \\
\hline & F97 & 0.8 & 22.6 & $6.9^{*}$ & $1.0-23.0$ & 0.36 \\
\hline & H97 & 4.7 & 31.8 & 16.6 & $2.4-60.8$ & 0.86 \\
\hline \multirow[t]{6}{*}{ DON } & L96a & 7.5 & 77.9 & $39.7 *$ & $5.9-102$ & 1.49 \\
\hline & L96b & 0.6 & 13.4 & $2.2 *$ & $0.1-18$ & 0.21 \\
\hline & L97a & 8.4 & 90.1 & $19.2 *$ & $8.2-84.6$ & 2.15 \\
\hline & L97b & 5.5 & 47.0 & $11.8^{*}$ & $1.1-38.2$ & 0.61 \\
\hline & F97 & 1.6 & 42.7 & 15.3 & $0-44.7$ & 0.80 \\
\hline & H97 & $\ldots$ & $\ldots$ & 8.0 & $0.7-27.0$ & 0.37 \\
\hline \multirow[t]{7}{*}{$\mathrm{HD}$} & L96a & 63.7 & 55.1 & 58.5 & $50-65$ & 0.36 \\
\hline & L96b & 65.5 & 60.8 & 61.0 & $50-70$ & 0.38 \\
\hline & St.P96 & 35.8 & 31.6 & 36.6 & $30-44$ & 0.37 \\
\hline & L97a & 62.0 & 60.0 & 60.3 & $52-66$ & 0.26 \\
\hline & L97b & 62.0 & 60.0 & 61.5 & $54-70$ & 0.34 \\
\hline & F97 & 71.0 & 65.0 & 67.4 & $59-76$ & 0.30 \\
\hline & H97 & 154.8 & 148.2 & 150.9 & $135-167$ & 0.52 \\
\hline \multirow[t]{5}{*}{ HT } & L96a & 96.1 & 75.4 & 87.4 & $70-110$ & 0.62 \\
\hline & L97a & 110.0 & 85.0 & $97.3^{*}$ & $78-123$ & 0.66 \\
\hline & L97b & 119.1 & 85.0 & 99.9 & $77-118$ & 0.62 \\
\hline & F97 & 99.2 & 72.6 & 86.1 & 63-104 & 0.63 \\
\hline & H97 & 119.1 & 95.4 & 107.2 & $81-129$ & 0.81 \\
\hline KP & F-GH98 & 21.8 & 96.4 & 79.2 & $5-99.9$ & 1.74 \\
\hline SA & F-GH98 & 3 & 1 & 2.1 & $1-3$ & 0.06 \\
\hline NN & F-GH98 & 7.49 & 7.78 & 7.32 & $3.23-9.05$ & 0.04 \\
\hline
\end{tabular}

$\mathrm{z} *$ = data $\log$ transformed when scanning for quantitative trait loci.
Correlation of traits. FHB severity was consistently and positively correlated with DON concentration (Table 2). The correlation coefficients ranged from 0.408 in environment $\mathrm{H} 97$ to 0.729 in L97a. Heading date and plant height also were positively correlated in all environments, except F97. However, the $r$ values between heading date and plant height were relatively low (with the highest $r$ values at $<0.5$ ). FHB severity was negatively correlated with heading date in four of the six environments (Table 2). A strong negative correlation between FHB severity and plant height also was observed in four of five environments where plant height measurements were taken. DON content showed a negative correlation with heading date in five of the six environments. DON content and plant height were also negatively correlated in four out of five environments (Table 2). In general, later heading and taller lines exhibited lower levels of FHB and DON.

There was no correlation between any of the three traits measured in the greenhouse, i.e., node length, spike angle, and kernel plumpness (data not shown). The correlation of these traits with FHB or DON was not determined because of the substantial differences between the environmental conditions of the greenhouse

\section{A}

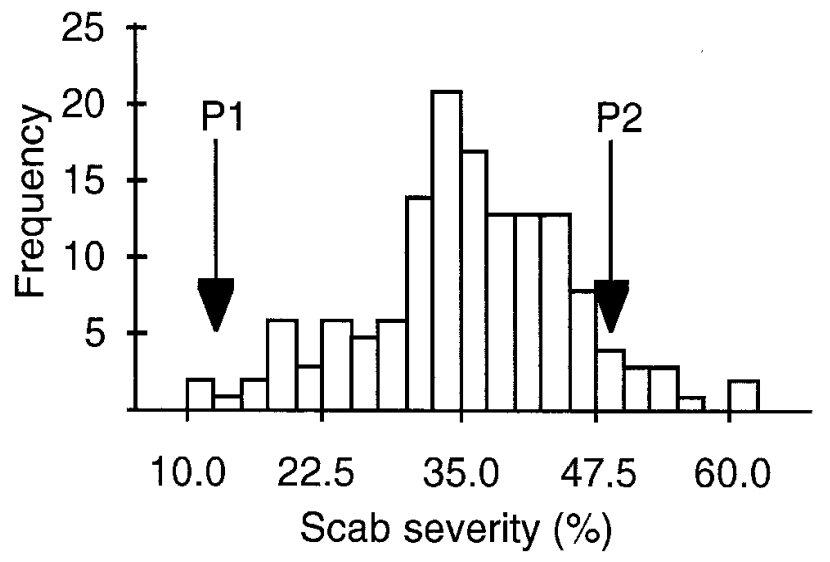

B

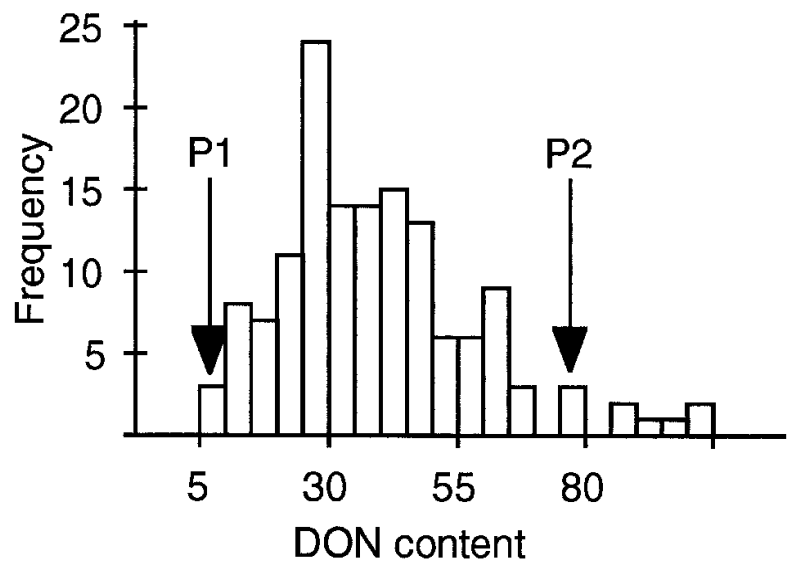

Fig. 2. Histograms showing the frequency distribution of A, Fusarium head blight severity (given as percent of infected kernels per spike) and $\mathbf{B}$, deoxynivalenol (DON) concentration of harvested grain (in parts per million) of doubled haploid progeny grown in the L96a environment. P1 refers to cv. Chevron, and P2 refers to $\mathrm{cv}$. Stander. 
where these traits were measured and the field where FHB severity and DON concentration were measured.

RFLP map. To systematically determine the chromosomal regions governing FHB resistance and the accumulation of DON in cv. Chevron, an RFLP framework map was constructed based on the cv. Chevron-Stander DH population. (Fig. 3). The map contains a total of 211 marker loci distributed among seven chromosomes and covers 1,026 centimorgans (cM). The identity of chromosomes was determined based on comparison with previously published barley maps $(4,8)$. The order of loci in this map is in agreement with those of Graner et al. (4), Heun et al. (5), and Kleinhofs et al. (8). The molecular marker map constructed for the cv. Chevron-Stander population provides good coverage of the barley genome, except for gaps of more than $30 \mathrm{cM}$ on chromosomes $4 \mathrm{H}$ and $5 \mathrm{H}$.

QTLs for FHB resistance, morphological, and agronomic traits. Eight QTLs for FHB severity were initially identified (Table 3 and Fig. 1). These were located on chromosomes 2HS, 3HS, 3HL, 5HS, 5HL, 6HL, and 7HS. The number of QTLs for FHB severity varied among environments from none in H97 to five in F97. The phenotypic variance explained by the multiple QTLs ranged from $30.0 \%$ in L96b to $43.9 \%$ in F97. The QTL on chromosome $2 \mathrm{HS}$ defined by Xbcd339c-Xbcd1407 was consistently detected in all five environments in North Dakota over the 2-year period. QTL analysis suggested the presence of more than one likelihood peak on this chromosome; thus, the "fix QTL" algorithm in Mapmaker/QTL was used to determine whether there was more than one QTL on this chromosome. Second-pass scanning revealed an additional QTL defined by $X b c d 307 b-X c d o 684 b$ on the long arm of chromosome $2 \mathrm{H}$ based on data from environment L97a. The phenotypic variance of both QTLs on 2HS and $2 \mathrm{HL}$ was $27.4 \%$; thus, $6.7 \%$ of this variance was explained by the QTL on 2HL. The two QTLs on chromosome $3 \mathrm{HL}$ are $\approx 30 \mathrm{cM}$ apart from each other. One QTL on chromosome 5HS was detected between Xmwg502 and Xbg739 based on data from environment L97a. After fixing this QTL on the short arm and rescanning the genome, a new QTL peak was mapped between Xbcd1449 and Xmwg533a on the long arm of chromosome $5 \mathrm{H}$. This QTL fell within the same confidence boundary interval as the only QTL peak detected for environment StP96.

Most of the alleles conditioning lower FHB severity came from cv. Chevron. These included the QTLs on chromosomes 2HS, 2HL, 3HS, 5HL, 6HL, and 7HS (Table 3). Except for the QTL on 2HL identified by second-pass scanning, all of these QTLs were detected in more than one environment. The susceptible parent $\mathrm{cv}$. Stander also had alleles that contributed to lower FHB severity. These were the QTLs on chromosomes 3HL and 5HS. All three of these QTLs were detected only in single environments, suggesting a large influence of the environment on the expression of these genes.

Nine QTLs for lower DON concentration were identified (Table 4 and Fig. 1). Eight of these overlapped with one for lower FHB severity. A QTL for lower DON on 2HL overlapped with the QTL for lower FHB on 2HL that was identified after second-pass scanning. The only QTL that did not overlap with a QTL for low FHB severity was on chromosome $1 \mathrm{H}$ between Xcdo431 and Xcmwg706. The number of QTLs identified in the environments ranged from two (F97) to five (L96a). The phenotypic variances explained by the multiple QTLs ranged from $15.1 \%$ in F97 to $60.7 \%$ in L97a (Table 4).

$\mathrm{Cv}$. Chevron was the parental source of the alleles for lower DON concentration on chromosomes $1 \mathrm{HL}, 2 \mathrm{HS}, 2 \mathrm{HL}, 3 \mathrm{HS}, 6 \mathrm{HL}$, and 7HS. The alleles on 3HL and 5HS for lower DON concentration came from cv. Stander and were detected in single environments only.

Four QTLs for heading date were detected initially on chromosomes 2HS, 3HL, 5HL, and 7HS (Table 5 and Fig. 1). All four overlapped with QTLs for either or both FHB severity and DON concentration (Fig. 1). The QTLs between Xbcd339c and
Xbcd1407 on chromosome 2HS and between Xwg789a and Xmwg836 on chromosome 7HS were detected in all trials (Table 5). Another QTL was identified on chromosome 2HS in environments L97b, StP96, and H97 after fixing the effect of the QTL between $X b c d 339 C$-Xbcd1407. The 2HS QTL was not associated with QTLs for lower FHB severity or DON concentration.

Nine QTLs for plant height were identified (Table 6 and Fig. 1). At least one QTL was present on every chromosome, except $1 \mathrm{H}$. The number of QTLs in the environments ranged from three to five. The phenotypic variance explained by the multiple QTLs ranged from $45.7 \%$ in F97 to $59.5 \%$ in L97b. Second-pass scanning for plant height QTLs was done on chromosomes 3HS and on 7HS which showed the presence of more than one likelihood peak. A new QTL on chromosome 3HLwas detected in environment L96a, which coincided with the QTL detected in environments L96b, L97b, F97, and HZ97. A new QTL also was identified on chromosome 7HL in environment L96a. This QTL was in the Xabg476a-Xcdo785e interval and was not detected in any other environment. Four plant height QTLs overlapped with QTLs for FHB severity and DON concentration (Fig. 1). These include those flanked by Xbcd339c-Xbcd1407 on $2 \mathrm{H}, \mathrm{Xabg} 316 \mathrm{a}$ Xcdo395 on $3 \mathrm{H}$, Xmwg502-Xbg739 on 5H, and Xcdo686Xcdo785d on 6HL.

One QTL was identified for kernel plumpness (Table 7 and Fig. 1) and was positioned in the Xwg719d-Xabc156e interval on chromosome $6 \mathrm{HL}$. This QTL gave a phenotypic variance of $10 \%$ and partially overlapped with a QTL for low FHB severity and DON (Fig. 1).

One QTL was identified for spike angle (Table 7 and Fig. 1) and was positioned in the Xcdo373-Xcdo684b interval on chromosome $2 \mathrm{HL}$. This QTL gave a phenotypic variance of $11 \%$ and partially overlapped with a QTL for low FHB severity and DON.

Five QTLs were detected for the number of nodes per centimeter of rachis (Table 7 and Fig. 1). These were located on chromosomes 1HL, 2HL, 3HL, 4HS, and 7HL. The multiple QTL model explained $45.8 \%$ of the phenotypic variation. The QTL on $2 \mathrm{HL}$ partially overlapped with QTLs for both FHB severity and DON, and the QTL on $1 \mathrm{H}$ partially overlapped with a QTL for DON (Fig. 1). The three other QTLs for the number of nodes per centimeter of rachis either partially $(3 \mathrm{H}$ and $7 \mathrm{H})$ or fully $(4 \mathrm{H})$ coincided with three different height QTLs.

TABLE 2. Correlation coefficients and $P$ values among Fusarium head blight (FHB) severity, deoxynivalenol (DON) concentration, heading date (HD), and plant height (HT) evaluated at Langdon, North Dakota (L96a, L96b, L97a, and L97b), Fargo, North Dakota (F97), and Hangzhou, China (HZ97)

\begin{tabular}{|c|c|c|c|c|}
\hline Experiment site & Trait & DON & $\mathrm{HD}$ & HT \\
\hline L96a & $\begin{array}{c}\text { FHB } \\
\text { DON } \\
\text { HD }\end{array}$ & $\begin{array}{c}0.675^{* * * *} \\
\ldots \\
\ldots\end{array}$ & $\begin{array}{c}-0.603 * * * * \\
-0.401 * * * * \\
\ldots\end{array}$ & $\begin{array}{c}-0.280 * * * \\
-0.243 * * \\
0.442 * * *\end{array}$ \\
\hline L96b & $\begin{array}{l}\text { FHB } \\
\text { DON }\end{array}$ & $\begin{array}{c}0.519 * * * * \\
\ldots\end{array}$ & $\begin{array}{l}-0.141 \\
-0.531 * * * *\end{array}$ & $\begin{array}{l}\text { NT } \\
\text { NT }\end{array}$ \\
\hline L97a & $\begin{array}{c}\text { FHB } \\
\text { DON } \\
\text { HD }\end{array}$ & $\begin{array}{c}0.729 \text { *** } \\
\ldots \\
\ldots\end{array}$ & $\begin{array}{c}-0.531 * * * * \\
-0.490 * * * * \\
\ldots\end{array}$ & $\begin{array}{c}-0.662 * * * * \\
-0.633 * * * * \\
0.371 * * *\end{array}$ \\
\hline L97b & $\begin{array}{c}\text { FHB } \\
\text { DON } \\
\text { HD }\end{array}$ & $\begin{array}{c}0.594 * * * * \\
\ldots \\
\ldots\end{array}$ & $\begin{array}{c}-0.572 * * * * \\
-0.435 * * * * \\
\ldots\end{array}$ & $\begin{array}{l}-0.452 * * * * \\
-0.442 * * * * \\
0.193 *\end{array}$ \\
\hline F97 & $\begin{array}{c}\text { FHB } \\
\text { DON } \\
\text { HD }\end{array}$ & $\begin{array}{c}0.639 * * * * \\
\ldots \\
\ldots\end{array}$ & $\begin{array}{r}-0.206^{*} \\
-0.139 \\
\ldots\end{array}$ & $\begin{array}{l}-0.389 * * * * \\
-0.373 * * * * \\
0.137\end{array}$ \\
\hline H97 & $\begin{array}{c}\text { FHB } \\
\text { DON } \\
\text { HD }\end{array}$ & $\begin{array}{c}0.408 * * * * * \\
\ldots \\
\ldots\end{array}$ & $\begin{array}{c}-0.112 \\
0.320^{* * * * *} \\
\ldots\end{array}$ & $\begin{array}{l}-0.011 \\
-0.157 \\
0.222 * *\end{array}$ \\
\hline
\end{tabular}

$\mathrm{z} *, * *, * * *$, and $* * * *=P \leq 0.05,0.01,0.001$, and 0.0001 , respectively. NT indicates data not taken at these locations. 
Second-pass scanning for FHB resistance QTLs. Statistically, the actual genetic variation of FHB resistance can be revealed, at least partially, by taking into consideration the associated traits and using them as covariables. A second-pass scanning of the genome for FHB severity QTLs was therefore conducted when the correlation of FHB severity to heading date or plant height was significant at $P=0.05$. This was performed by using the algorithm in Mapmaker/QTL to fix the effect of the associated trait as described previously.
Second-pass scanning of QTLs for FHB severity using heading date or plant height as covariables resulted in the disappearance of the QTL between $X b c d 339 c-X b c d 1407$ on $2 \mathrm{HS}$ in environments L96a, L96b, L97a, and L97b, but not in F97. The QTL between Xbcd512c-Xcdol05 on 3H disappeared in environment L96a, and the QTL between Xmwg36b-Xmwg836 on $7 \mathrm{H}$ disappeared in environments L96b and L97b. The 5HS QTL in L97a and the 5HL QTLs in F97 and StP96 also disappeared after second-pass scanning.
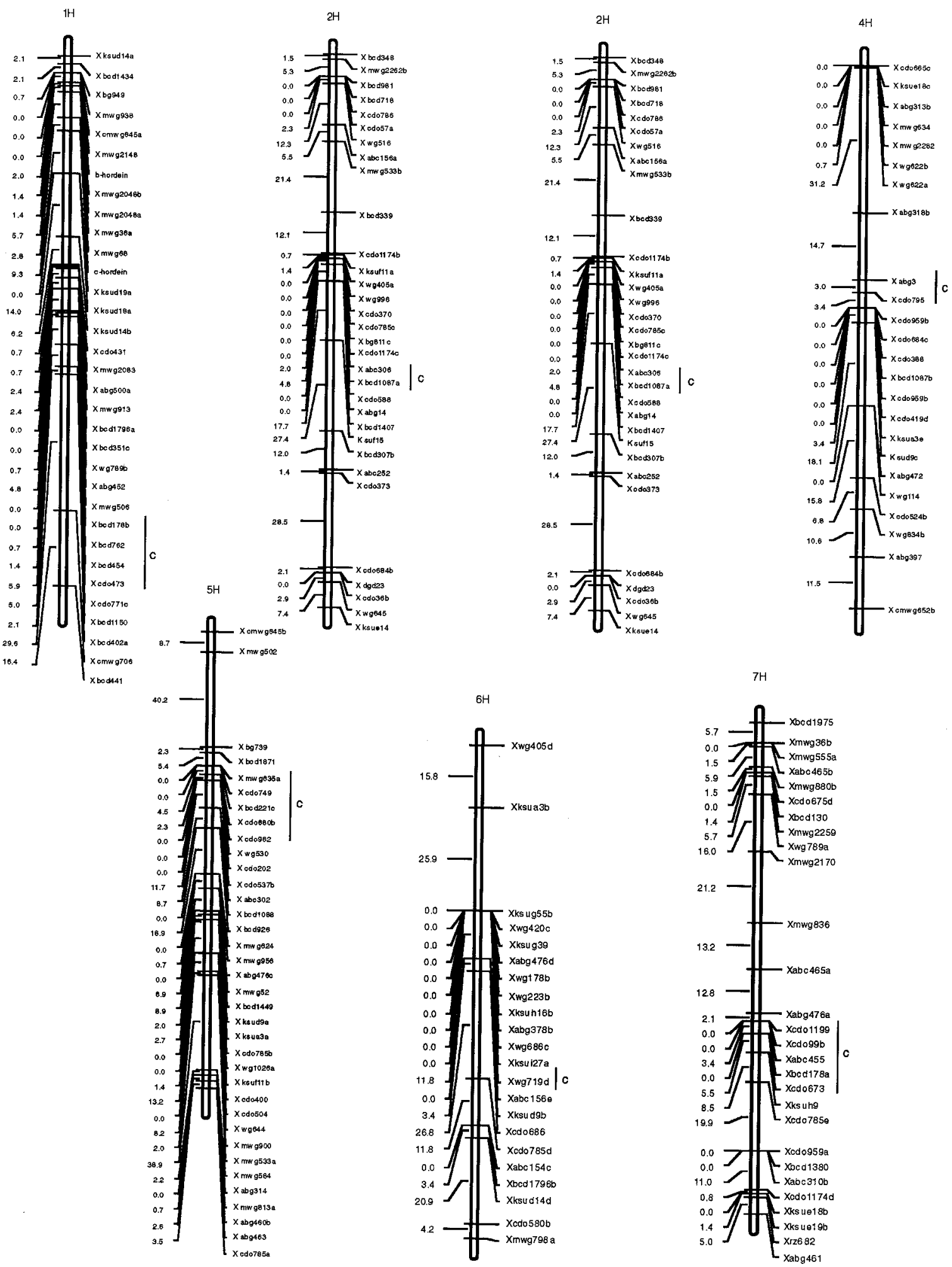

Fig. 3. Restriction fragment length polymorphism map constructed in a doubled haploid population derived from a cross between cvs. Chevron and Stander. Genetic distances in centimorgans (cM) are calculated based on Kosambi function (9). The upper portion is the short arm and the lower portion is the long arm. Approximate positions of centromeres are shown by the notation $\mathrm{C}$. 
Second-pass scanning revealed two new QTLs for FHB severity. One was detected in the L97b field trial, and it was mapped between Xcmwg706 and Xbcd441 on chromosome 1H (Fig. 1). This QTL is linked to QTLs for DON concentration and number of nodes per centimeter of rachis (Fig. 1). The allele of this QTL conferring lower FHB severity came from cv. Chevron. A second QTL was detected in environments L96a, L97a, and L97b, and it was mapped between Xcdo959b and Xabg472 on chromosome $4 \mathrm{H}$ (Fig. 1). This allele also came from cv. Chevron.

\section{DISCUSSION}

This paper reports the genetic mapping of QTLs associated with relatively low FHB severity and DON content in cv. Chevron, the most resistant six-rowed accession to FHB identified to date. QTLs for other morphological traits (heading date, plant height, spike angle, kernel plumpness, and kernel density) were also mapped to assess their potential effect on the level of FHB observed.

DH progeny were produced from the cv. Chevron-Stander cross to allow replications to be evaluated in different locations and over several years on the same set of homozygous genotypes. This is an important aspect in mapping determinants of FHB resistance, because the expression can be extremely variable in the field. FHB and DON data were obtained on DH progeny from seven environments in four locations where artificial inoculation and overhead irrigation were used to facilitate disease development over a 2-year period. Despite the use of DH progeny and replicated experiments in the same location, differences were observed among the trials for the number of QTL and genomic location of QTLs for the two FHB traits. Variability in the pathogen isolates is an unlikely explanation for the differences observed among the field experiments because the same isolates of $F$. graminearum were used in the inoculations and likely predominated in the nurs- eries at all North Dakota locations. The widest possible isolate differences would be those in China compared to the United States. However, even in the case of virulence differences in the pathogen among locations, no significant cultivar-isolate interactions have been detected indicating the lack of gene for gene interactions of host and pathogen (30). It is more likely that the variation in identified QTL results from genotype-environment interaction.

Nine QTLs each were associated with lower FHB severity and DON concentration and eight were shared between the two traits. This result is in agreement with previously reported positive correlations between these two traits $(2,35)$. The lack of coincidence of the remaining one QTL for FHB severity and for DON concentration may be attributed to the variability of expression in different environments. It is also possible that the QTL for low FHB severity on chromosome 1 may represent a gene for Type III resistance. This type of resistance has been described in wheat and is thought to contribute to a reduction in the synthesis of DON or the rapid breakdown of DON (18). Controlled greenhouse inoculation tests will be needed to confirm the possible presence of a QTL contributing to putative Type III resistance.

Seven QTLs for FHB and six QTLs for DON coincided with QTLs for either or both heading date and plant height (Fig. 1). This agrees with the negative correlation observed between lower FHB severity or DON concentration and heading date or plant height in some of the trials (Table 2). The overlap between QTLs for FHB resistance and those for heading date and plant height could indicate one of two things. One, there may be linkage between genes for FHB resistance and genes for heading date or plant height. A second possibility is that the observed resistance may be a result of pleiotropic effects. Heading date and height are important factors in the development of FHB on plants. Although adequate amounts of inoculum and overhead irrigation were pro-

TABLE 3. Quantitative trait loci (QTL) for Fusarium head blight (FHB) severity, and chromosome location, percent phenotypic variance (VE), logarithm of odds (LOD), and parental source of the resistant allele for each QTL in each environment ${ }^{\mathrm{z}}$

\begin{tabular}{|c|c|c|c|c|c|c|c|c|c|c|c|c|c|c|}
\hline \multirow[b]{2}{*}{ QTL marker interval } & \multirow[b]{2}{*}{ Chromosome } & \multicolumn{2}{|c|}{ L96a } & \multicolumn{2}{|c|}{ L96b } & \multicolumn{2}{|c|}{ L97a } & \multicolumn{2}{|c|}{ L97b } & \multicolumn{2}{|c|}{ F97 } & \multicolumn{2}{|c|}{ StP96 } & \multirow[b]{2}{*}{ Source } \\
\hline & & LOD & VE & LOD & VE & LOD & VE & LOD & VE & LOD & VE & LOD & VE & \\
\hline Xbcd339c-Xbcd1407 & $2 \mathrm{HS}$ & 4.58 & 13.7 & 4.26 & 12.8 & 4.08 & 20.7 & 3.85 & 11.8 & 5.13 & 15.2 & $\ldots$ & $\ldots$ & $\mathrm{C}$ \\
\hline Xbcd307b-Xcdo684b & $2 \mathrm{HL}$ & $\ldots$ & $\ldots$ & $\ldots$ & $\ldots$ & 5.63 & 27.4 & & & $\ldots$ & & $\ldots$ & $\ldots$ & $\mathrm{C}$ \\
\hline Xbcd512c-Xcdo105 & $3 \mathrm{HL}$ & 2.43 & 7.5 & $\ldots$ & $\ldots$ & $\ldots$ & $\ldots$ & $\ldots$ & $\ldots$ & $\ldots$ & $\ldots$ & $\ldots$ & $\ldots$ & $\mathrm{S}$ \\
\hline Xwg420b-Xabc172 & $3 \mathrm{HL}$ & $\ldots$ & $\ldots$ & $\ldots$ & $\ldots$ & $\ldots$ & $\ldots$ & $\ldots$ & $\ldots$ & 3.01 & 9.5 & $\ldots$ & $\ldots$ & $\mathrm{S}$ \\
\hline Xmwg502-Xbg739 & $5 \mathrm{HS}$ & $\ldots$ & $\ldots$ & $\ldots$ & $\ldots$ & 3.30 & 17.3 & $\ldots$ & $\ldots$ & $\ldots$ & $\ldots$ & $\ldots$ & $\ldots$ & $\mathrm{S}$ \\
\hline Xmwg36b-Xmwg836 & $7 \mathrm{HS}$ & 6.18 & 21.3 & 3.11 & 12.8 & $\ldots$ & $\ldots$ & 4.39 & 13.2 & $\ldots$ & $\ldots$ & $\ldots$ & $\ldots$ & $\mathrm{C}$ \\
\hline Multi-QTL model & $\ldots$ & 15.0 & 41.3 & 9.21 & 30.0 & 7.43 & 34.7 & 14.9 & 39.5 & 17.0 & 43.9 & $\ldots$ & $\ldots$ & $\ldots$ \\
\hline
\end{tabular}

${ }^{\mathrm{z}}$ Loci whose LOD and VE values are shown in plain text represent QTLs detected using the entire map: those in bold are QTLs detected after fixing the effect of the main QTL on the specific chromosome and rescanning the genome for QTLs. The phenotypic variance of these QTLs include the effect of both the original and new QTLs. VE values for the multi-QTL model do not include the VE for QTLs detected after second-pass scanning. $\mathrm{C}=\mathrm{cv} . \mathrm{Chevron}$ and $\mathrm{S}=\mathrm{cv}$. Stander.

TABLE 4. Quantitative trait loci (QTLs) for deoxynivalenol (DON) concentration, and chromosome location, logarithm of odds (LOD), percent phenotypic variance (VE), and parental source of the resistant allele for each QTL in each environment ${ }^{\mathrm{z}}$

\begin{tabular}{|c|c|c|c|c|c|c|c|c|c|c|c|c|}
\hline \multirow[b]{2}{*}{ QTL marker interval } & \multirow[b]{2}{*}{ Chromosome } & \multicolumn{2}{|c|}{ L96a } & \multicolumn{2}{|c|}{ L96b } & \multicolumn{2}{|c|}{ L97a } & \multicolumn{2}{|c|}{ L97b } & \multicolumn{2}{|c|}{ F97 } & \multirow[b]{2}{*}{ Source } \\
\hline & & LOD & VE & LOD & VE & LOD & VE & LOD & VE & LOD & VE & \\
\hline Xcdo431- Xcmwg706 & $1 \mathrm{HL}$ & 2.08 & 6.5 & 2.29 & 11.1 & $\ldots$ & .. & & & & & $\mathrm{C}$ \\
\hline Xbcd339c-Xbcd1407 & $2 \mathrm{HS}$ & 4.92 & 14.8 & 7.25 & 21.6 & $\ldots$ & $\ldots$ & 6.9 & 19.9 & 2.66 & 9.6 & $\mathrm{C}$ \\
\hline Xabg316a-Xcdo395 & $3 \mathrm{HS}$ & $\ldots$ & $\ldots$ & $\ldots$ & $\ldots$ & $\ldots$ & $\ldots$ & $\ldots$ & $\ldots$ & 2.64 & 8.5 & $\mathrm{C}$ \\
\hline Xbcd512c-Xcdo105 & $3 \mathrm{HL}$ & 2.27 & 7.8 & $\ldots$ & $\ldots$ & $\ldots$ & $\ldots$ & $\ldots$ & $\ldots$ & $\ldots$ & $\ldots$ & $\mathrm{S}$ \\
\hline Xwg420b-Xabc172 & $3 \mathrm{HL}$ & $\ldots$ & $\ldots$ & 2.4 & 8.2 & $\ldots$ & $\ldots$ & $\ldots$ & $\ldots$ & $\ldots$ & $\ldots$ & $\mathrm{S}$ \\
\hline Multi-QTL model & $\ldots$ & 13.87 & 37.1 & 18.22 & 48.1 & 14.19 & 60.7 & 13.5 & 36.3 & 4.58 & 15.1 & $\ldots$ \\
\hline
\end{tabular}

${ }^{\mathrm{z}} \mathrm{S}=\mathrm{cv}$. Stander and $\mathrm{C}=\mathrm{cv}$. Chevron. 
vided throughout the heading period of the DH lines, some late heading lines may have escaped the most favorable period for infection in the field and therefore had lower FHB. Shorter plants usually have higher disease levels because they are closer to the Fusarium inoculum originating from the soil surface (18). Moreover, short-statured plants often stay wet longer because of their close proximity to the sometimes saturated soil surface. The possible confounding effects of traits like heading date and plant height on FHB development are difficult to resolve in the field. To determine their true effect on FHB severity, the DH population should be evaluated in uniform and strictly controlled inoculation studies in the greenhouse or growth chamber.

The "fix-QTL" algorithm in Mapmaker/QTL was used to perform a second-pass scanning for QTLs contributing to FHB severity when heading date or plant height were significantly $(P \leq 0.05)$ correlated with FHB severity. This analysis is usually applied when some portion of a phenotypic variance can be explained by another trait (M. Daly, personal communication). Second-pass scanning removes the part of the variance that is explained by the associated trait so the genetic basis becomes more evident. When heading date or height was used as a covariable in second-pass scanning for QTLs for FHB severity, the QTLs on chromosomes $2 \mathrm{HS}, 5 \mathrm{HS}, 5 \mathrm{HL}$, and 7HS disappeared in some of the field trials. Interestingly, two new QTLs that were not detected in the first-pass scanning were identified, $x c m w g 701-x b c d 441$ on chromosome $1 \mathrm{H}$ and Xcdo959b-Xabg472 on chromosome 4H (Fig. 1).

FHB resistance in cv. Chevron was also mapped genetically by de la Pena et al. (2) in a parallel study conducted in Minnesota. In that study, cv. Chevron was crossed with a susceptible elite breeding line M69 and 101 $\mathrm{F}_{4: 7}$ families were used in mapping. Using a map consisting of 96 loci, eight QTLs for FHB severity and two QTLs for DON content were identified. Both QTLs for
DON content overlapped with two of the QTLs for FHB severity. All alleles conditioning lower FHB severity were from cv. Chevron. The eight QTL intervals for FHB severity include abg452abg74 on $1 \mathrm{H}, a b c 311-m w g 858$ on $2 \mathrm{H}, m w g 887-a b c 306$ on $2 \mathrm{H}$, abg14-abg619 on $2 \mathrm{H}$, abc171-cdo395 on $3 \mathrm{H}$, abg705-abc303 on $4 \mathrm{H}, c d o 400-c d o 59$ on $5 \mathrm{H}$, and $m w g 530-m w g 564$ on $7 \mathrm{H}$. Seven of these eight FHB QTL intervals are in the same chromosome region as five QTL intervals for FHB or DON detected in this study based on the presence of a common marker in each QTL interval. For example, the markers $a b g 452, a b c 306, a b g 14$, and $a b c 171$ are found in the QTL intervals on $1 \mathrm{H}$ (for DON), $2 \mathrm{HS}, 2 \mathrm{HS}$, and 3HS, respectively. Two other QTL intervals identified by de la Pena et al. (mwg530-mwg564 and abg705-abc303) contain markers on the barley consensus map that are linked to at least one RFLP marker within the FHB resistance QTL intervals on chromosomes $7 \mathrm{H}$ and $4 \mathrm{H}$, respectively (2). Only one of the eight QTLs identified by de la Pena et al. (on chromosome $2 \mathrm{H}$ between $a b c 311-m w g 858)$ (2) did not have a corresponding QTL interval in this study.

Only one of the eight QTLs for FHB severity identified by de la Pena et al. (abc311-mwg858 on 2H) (2) overlapped with a QTL for heading. This was in contrast to this study where four of nine QTLs for FHB overlapped with a QTL for heading. The different results obtained between the two studies may be due to a number of factors including genotype-environment interactions, differences in the genotypes of the susceptible parents, and perhaps the type of population (DH vs. recombinant inbred lines) used.

It may be concluded from the results of these two studies that it is likely the QTL on chromosome $2 \mathrm{H}$ between $\mathrm{Xbcd339c}$ and Xbcd1407 does not contain genes for FHB resistance per se because in both studies this QTL was associated with a heading date QTL. This region is also known to contain the Vrsl locus for two

TABLE 5. Quantitative trait loci (QTLs) for heading date, and chromosome location, percent phenotypic variance (VE), logarithm of odds (LOD), and parental source of the allele contributing to late heading for each QTL in each environment ${ }^{2}$

\begin{tabular}{|c|c|c|c|c|c|c|c|c|c|c|c|c|c|c|c|c|}
\hline \multirow[b]{2}{*}{ QTL marker interval } & \multirow[b]{2}{*}{ Chromosome } & \multicolumn{2}{|c|}{ L96a } & \multicolumn{2}{|c|}{ L96b } & \multicolumn{2}{|c|}{ L97a } & \multicolumn{2}{|c|}{ L97b } & \multicolumn{2}{|c|}{ F97 } & \multicolumn{2}{|c|}{ StP96 } & \multicolumn{2}{|c|}{$\mathrm{HZ}$} & \multirow[b]{2}{*}{ Source } \\
\hline & & LOD & VE & LOD & VE & LOD & VE & LOD & VE & LOD & VE & LOD & VE & LOD & VE & \\
\hline Xbcd339c-Xbcd1407 & $2 \mathrm{HS}$ & 5.42 & 16.0 & 4.17 & 16.7 & 5.26 & 15.6 & 5.22 & 15.5 & 7.18 & 20.6 & 2.53 & 11.8 & 4.00 & 12.3 & S \\
\hline Xmwg533b-Xmwg584 & $5 \mathrm{HL}$ & & & & & 2.71 & 16.5 & & & & & & & 3.08 & 9.6 & $\mathrm{C}$ \\
\hline Xwg789a-Xmwg836 & 7HS & 8.94 & 34.3 & 2.28 & 11.2 & 5.92 & 20.7 & 3.77 & 11.4 & 5.52 & 21.5 & 12.56 & 59.0 & 8.69 & 33.0 & $\mathrm{~S}$ \\
\hline
\end{tabular}

${ }^{\mathrm{z}}$ Loci whose LOD and VE values are shown in plain font represent QTLs detected using the entire map; those in bold are QTLs detected after fixing the effect of the main QTL on the specific chromosome and rescanning the genome for QTLs. The phenotypic variance of these QTLs include the effect of both the original and new QTLs. S = cv. Stander; C = cv. Chevron. The VE values for the multi-QTL model do not include the VE for QTLs detected after second-pass scanning.

TABLE 6. Quantitative trait loci (QTLs) for plant height, and chromosome location, logarithm of odds (LOD), percent phenotypic variance (VE), and parental source of the allele contributing to increased plant height for each QTL in each environment ${ }^{2}$

\begin{tabular}{|c|c|c|c|c|c|c|c|c|c|c|c|c|}
\hline \multirow[b]{2}{*}{ QTL marker interval } & \multirow[b]{2}{*}{ Chromosome } & \multicolumn{2}{|c|}{ L96a } & \multicolumn{2}{|c|}{ L96b } & \multicolumn{2}{|c|}{ L97b } & \multicolumn{2}{|c|}{ F97 } & \multicolumn{2}{|c|}{$\mathrm{HZ}$} & \multirow[b]{2}{*}{ Source } \\
\hline & & LOD & VE & LOD & VE & LOD & $\mathrm{VE}$ & LOD & $\mathrm{VE}$ & LOD & VE & \\
\hline$X b c d 339 c-X b c d 1407$ & $2 \mathrm{HS}$ & 2.81 & 10.4 & $\ldots$ & $\ldots$ & $\ldots$ & $\ldots$ & $\ldots$ & $\ldots$ & 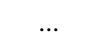 & 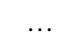 & $\mathrm{S}$ \\
\hline Xabg316a-Xcdo395 & $3 \mathrm{HS}$ & 3.31 & 10.2 & $\ldots$ & $\ldots$ & . & $\ldots$ & $\ldots$ & $\ldots$ & 2.71 & 8.5 & $\mathrm{~S}$ \\
\hline Xabg318b-Xabg3 & $4 \mathrm{HS}$ & 2.42 & 9.1 & 2.29 & 8.4 & $\ldots$ & $\ldots$ & $\ldots$ & $\ldots$ & $\ldots$ & $\ldots$ & $\mathrm{C}$ \\
\hline Xabg472-Xcdo524b & $4 \mathrm{HL}$ & $\ldots$ & $\ldots$ & $\ldots$ & $\ldots$ & 2.41 & 8.9 & $\ldots$ & $\ldots$ & 1.68 & 5.1 & $\mathrm{C}$ \\
\hline Xmwg502-Xbg739 & $5 \mathrm{HS}$ & 1.65 & 8.0 & 2.86 & 14.8 & 3.9 & 21.6 & 3.39 & 17.9 & $\ldots$ & & $\mathrm{C}$ \\
\hline Xmwg836-Xabg476a & $7 \mathrm{HS}$ & 5.36 & 17.6 & 5.42 & 18.1 & 5.36 & 20.7 & 5.28 & 20.0 & 9.89 & 31.3 & $\mathrm{~S}$ \\
\hline Xabg476a-Xcdo785e & 7HL & 3.24 & 8.2 & $\ldots$ & $\ldots$ & $\ldots$ & $\ldots$ & $\ldots$ & $\ldots$ & $\ldots$ & $\ldots$ & $\mathrm{S}$ \\
\hline Multi-QTL model & $\ldots$ & 17.0 & 48.2 & 15.88 & 50.7 & 18.64 & 59.5 & 15.06 & 45.7 & 20.78 & 53.0 & $\ldots$ \\
\hline
\end{tabular}

${ }^{\mathrm{z}}$ Loci whose LOD and VE values are shown in plain text represent QTLs detected using the entire map; those in bold are QTLs detected after fixing the effect of the main QTL on the specific chromosome and rescanning the genome for QTLs. The phenotypic variance of these QTLs include the effect of both the original and new QTLs. The VE values for the multi-QTL model do not include the VE for QTLs detected after second-pass scanning. S $=\mathrm{cv}$. Stander and C $=$ cv. Chevron. 
six-row type that is linked to a heading date QTL with a large effect (7). Of the four other QTLs that disappeared after secondpass scanning, the intervals Xbcd1449-Xmwg533a on chromosome 5H and Xmwg789a-Xmwg836 on chromosome 7H were not associated with late heading in de la Pena et al.'s (2) study and need to be tested further. The two other QTLs for lower FHB severity (Xbc512c-Xcdo105 on 3HL and Xmwg502-Xbg739 on $5 \mathrm{H})$ were from the susceptible parent cv. Stander. Although cv. Stander is early heading and short, these QTL intervals contained cv. Stander alleles that contributed to later heading and increased height.

In this study, FHB QTLs with large phenotypic variances (for example, Xbcd339c-Xbcd1407 on 2HS, Xmwg502-Xbg739 on 5HS, and Xmwg36b-Xmwg836 on 7H) were associated with QTLs for heading date and plant height; thus, it is possible that QTLs with major effects for FHB resistance per se do not exist specifically in cv. Chevron. The QTL intervals Xcmwg706-Xbcd441 on chromosome $1 \mathrm{H}, X b c d 307 b-X c d o 684 b$ on chromosome $2 \mathrm{H}$, and $X c d o 959 b-X a b g 472$ on chromosome $4 \mathrm{H}$ were not associated with heading date or plant height QTLs and may be useful for markerassisted selection. However, these QTLs had relatively small effects in this study. A critical future study would be to test the effectiveness of these QTLs in a background without the QTLs for late heading and tall plant height.

QTLs for other morphological traits (i.e., spike angle, kernel plumpness, number of nodes per centimeter of rachis) were mapped in this study and partially overlapped with QTLs for FHB resistance or DON content. Zhu et al. (35) mapped QTLs for FHB resistance and other morphological traits including inflorescence structure, average distance between seeds within the inflorescence, and lateral floret size in a DH population derived from the two-rowed parent cvs. Gobernadora and CMB643. They found that all but two of the FHB resistance QTLs identified coincided with QTLs for one or more of these morphological traits. Zhu et al. (35) concluded that plant architectural features are important determinants in the development of FHB on barley.

Waldron et al. (32) recently reported mapping of QTLs for FHB resistance in wheat. Five QTLs were detected: one each on chromosomes $2 \mathrm{~A}, 3 \mathrm{~B}$, and $4 \mathrm{~B}$ and two on $6 \mathrm{~B}$. The percent phenotypic variance explained by these QTLs ranged from 3.9 to $15.4 \%$. Their study differed from all the studies done in barley thus far in that their experiments were conducted in the greenhouse rather than in the field.

The information obtained in this study and other FHB resistance mapping studies in barley $(2,32)$ should be useful for breeding because it provides breeders with information on QTLs to select for or against. Marker-assisted selection for this trait will overcome the difficulties of FHB assessments associated with the variable expression of resistance in different environments. The findings from this study that the QTLs contributing major effects on FHB resistance overlap with QTLs for late heading and increased plant height in cv. Chevron highlights another important

TABLE 7. Quantitative trait loci (QTLs) for kernel plumpness (KP), spike angle (SA), and number of nodes per centimeter of rachis (NN), and chromosome location, logarithm of odds (LOD), and percent phenotypic variance $(\mathrm{VE})$

\begin{tabular}{llccr}
\hline Trait & QTL marker interval & Chromosome & LOD & VE \\
\hline KP & Xwg719d-Xabc156e & 6HL & 2.83 & 9.9 \\
SA & Xcdo373-Xcdo684b & 2HL & 2.74 & 11.1 \\
NN & Xbcd402a-Xmwg706 & 1HL & 2.19 & 7.0 \\
& Xbcd307b-Xabc252 & 2HL & 4.38 & 13.5 \\
& Xwg940-Xabg4 & 3HL & 2.64 & 14.4 \\
& Xabg318b-Xabg3 & 4HS & 2.15 & 8.3 \\
& Xcdo673-Xdgh9 & 7HL & 2.16 & 7.1 \\
& Multi-QTL model & $\ldots$ & 15.21 & 45.8 \\
\hline
\end{tabular}

contribution of QTL mapping in breeding. Based on phenotypic selection alone, a breeder using cv. Chevron as the resistant parent may select for resistance for several generations but may not be able to produce a resistant variety that also carries the desired traits of early heading, short stature, plump kernels, or six-rowed type.

\section{ACKNOWLEDGMENTS}

This project was partially supported by the North American Barley Mapping Project (NABGMP), American Malting Barley Association, Busch Agricultural Resources Inc., and Hatch Funds 644. We thank D. Rasmusson for conducting the field experiments in St. Paul, MN in 1996, E. Frost for technical assistance in the laboratory experiments, J. Menert for assistance with the greenhouse inoculations and screening, and P. Byrne for his comments on the manuscript.

\section{LITERATURE CITED}

1. Chen, F., and Hayes, P. M. 1989. A comparison of Hordeum bulbosummediated haploid production efficiency in barley using in vitro floret and tiller culture. Theor. Appl. Genet. 77:701-704.

2. de la Pena, R. C., Smith, K. P., Capettini, F., Rasmusson, D. C., GalloMeagher, M., Dill-Macky, R., Somers, D. A., and Muehlbauer, G. J. 1999. Quantitative trait loci associated with resistance to Fusarium head blight and kernel discoloration in barley. Theor. Appl. Genet. 99:561-569.

3. Gill, K. S., Lubbers, E. L., Gill, B. S., Raupp, W. J., and Cox, T. S. 1991. A genetic linkage map of Triticum tauschii (DD) and its relationship to the D genome of bread wheat (AABBDD). Genome 34:362-374.

4. Graner, A., Jahoor, A., Schondelmaier, J., Siedler, H., Pillen, K., Fischbeck, G., Wenzel, G., and Hermann, R. G. 1991. Construction of an RFLP map of barley. Theor. Appl. Genet. 83:250-256.

5. Heun, M., Kennedy, A. E., Anderson, J. A., Lapitan, N. L. V., Sorrells, M. E., and Tanksley, S. D. 1991. Construction of a restriction fragment length polymorphism map for barley. Genome 34:437-447.

6. Joffe, A. 1986. Fusarium Species: Their Biology and Toxicology. John Wiley \& Sons, New York.

7. Kjaer, B., and Jensen, J. 1996. Quantitative trait loci for grain yield and yield components in a cross between a six-rowed and a two-rowed barley. Euphytica 90:39-48.

8. Kleinhofs, A., Kilian, A., Saghai Maroof, M. A., Biyashev, R. M., Hayes, P. M., Chen, F. Q., Lapitan, N., Fenwick, A., Blake, T. K., Kanazin, V., Ananiev, E., Dahleen, L., Kudrna, D., Bollinger, J., Knapp, S. J., Liu, B., Sorrells, M., Heun, M., Franckowiak, J. D., Hoffman, D., Skadsen, R., and Steffenson, B. J. 1993. A molecular, isozyme and morphological map of the barley (Hordeum vulgare) genome. Theor. Appl. Genet. 86:705-712.

9. Kosambi, D. D. 1944. The estimation of map distances from recombination values. Ann. Eugen. 12:172-175.

10. Lander, E. S., and Botstein, D. 1989. Mapping Mendelian factors underlying quantitative traits using RFLP linkage maps. Genetics 121:185-199.

11. Lander, E. S., Green, P., Abrahamson, J., Barlow, A., Daly, M. J., Lincoln, S. E., and Newburg, L. 1987. MAPMAKER: An interactive computer package for constructing primary linkage maps of experimental and natural populations. Genomics 1:174-181.

12. Lincoln, S., Daly, M., and Lander, E. 1992. Mapping genes controlling quantitative traits with Mapmaker/QTL 1.1. 2nd ed. Whitehead Inst. Tech. Rep.

13. Linde-Laursen, I., Heslop-Harrison, J. S., Shepherd, K. W., and Taketa, S. 1997. The barley genome and its relationship with the wheat genomes: A survey with an internationally agreed recommendation for barley chromosome nomenclature. Hereditas 126:1-16.

14. Lynch, M., and Wash, B. 1997. Genetics and analysis of quantitative traits. Sinauer Associates Inc., Sunderland, MA.

15. Ma, Z.-Q., and Sorrells, M. E. 1995. Genetic analysis of fertility restoration in wheat using restriction fragment length polymorphisms. Crop Sci. 35:1137-1143.

16. Mathre, D. E. 1997. A Compendium of Barley Diseases. The American Phytopathological Society, St. Paul, MN.

17. McMullen, M. D., and Louie, R. 1989. The linkage of molecular markers to a gene controlling the symptom response in maize to maize dwarf mosaic virus. Mol. Plant-Microbe Interact. 2:309-314.

18. Mesterhazy, A. 1995. Types and components of resistance to Fusarium head blight of wheat. Plant Breeding 114:377-386.

19. Parry, D. W., Jenkinson, P., and McLeod, L. 1995. Fusarium ear blight (scab) in small grain cereals: A review. Plant Pathol. 44:207-238.

20. Paterson, A. H., Lander, E. S., Hewitt, J. D., Peterson, S., Lincoln, S. E., and Tanksley, S. D. 1988. Resolution of quantitative traits into 
Mendelian factors by using a complete linkage map of restriction fragment length polymorphisms. Nature 335:721-726.

21. Prom, L. K., Horsley, R. D., Steffenson, B. J., and Schwarz, P. B. 1999. Development of Fusarium head blight and accumulation of deoxynivalenol in barley sampled at different growth stages. J. Am. Soc. Brew. Chem. 57:60-63.

22. Prom, L. K., Steffenson, B. J., Salas, B., Fetch, T. G., Jr., and Casper, H. H. 1997. Barley accessions resistant to Fusarium head blight and the accumulation of deoxynivalenol. Cereal Res. Commun. 25:807-808.

23. Schwarz, P. B., Beattie, S., and Casper, H. W. 1996. Relationship between Fusarium infestation of barley and the gushing potential of malt. J. Inst. Brew. 102:93-96.

24. Scott, P. M. 1990. Triothecenes in grains. Cereal Foods World 35:661-666.

25. Shands, R. G. 1939. Chevron, a barley variety resistant to stem rust and other diseases. Phytopathology 29:209-211.

26. Sherman, J. D., Fenwick, A. L., Namuth, D. M., and Lapitan, N. L. V. 1995. A barley RFLP map: Alignment of three barley maps and comparisons to Gramineae species. Theor. Appl. Genet. 91:681-690.

27. Steffenson, B. J. 1998. Fusarium head blight of barley: Epidemics, impact, and breeding for resistance. Master Brew Assoc. Am. Tech. Q. 35:177-183.

28. Tacke, B. K., and Casper, H. H. 1996. Determination of deoxynivalenol in wheat, barley, and malt by column cleanup and gas chromato- graphy with electron capture detection. J. Assoc. Off. Anal. Chem. 79:472-475.

29. Takeda, K., and Heta, H. 1989. Establishing the testing methods and a search for the resistant varieties to Fusarium head blight in barley. Jpn. J. Breed. 39:203-216.

30. Takeda, K., and Kanatani, R. 1991. Host-pathogen relationship in barley scab. J. Breed. 41:641-650.

31. Vivar, H. 1987. A new barley variety from China. Rachis 6:17-19.

32. Waldron, B. L., Moreno-Sevilla, B., Anderson, J. A., Stack, R. W., and Frohberg, R. C. 1999. RFLP mapping of QTL for Fusarium head blight resistance in wheat. Crop Sci. 39:805-811.

33. Wang, Y. Z., and Miller, J. D. 1988. Screening techniques and sources of resistance to Fusarium head blight. Pages 239-250 in: Wheat Production Constraints in Tropical Environments. International Maize and Wheat Improvement Center, Mexico, D.F.

34. Zadoks, J. C., Chang, T. T., and Konzak, C. F. 1974. A decimal code for the growth stages of cereals. Weed. Res. 14:415-421.

35. Zhu, H., Gilchrist, L., Hayes, P., Kleinhofs, A., Kudrna, D., Liu, Z., Steffenson, B., Prom, L., Toojinda, T., and Vivar, H. 1999. Function follows form: Coincident QTLs are determinants of plant architecture traits and Fusarium head blight (FHB) resistance in a doubled haploid population of barley. Theor. Appl. Genet. 99:1221-1232.

\section{Erratum}

\section{Vol. 90, No. 10, 2000}

In the manuscript entitled "Mapping of Quantitative Trait Loci for Fusarium Head Blight Resistance in Barley" by Z. Ma, B. J. Steffenson, L. K. Prom, and N. L. V. Lapitan (Phytopathology 90:1079-1088), chromosome 3 is missing in Figure 3 of the article and is included below.

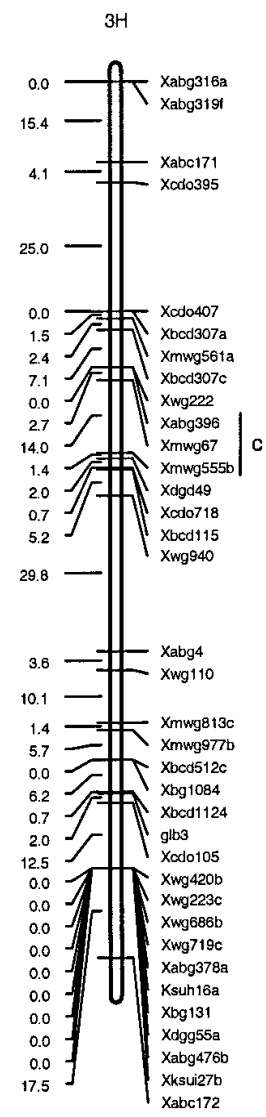

